\title{
Stability and universal encapsulation of epitaxial Xenes
}

\author{
Alessandro Molle, (iD *a Gabriele Faraone, ${ }^{\text {ab }}$ Alessio Lamperti, ${ }^{a}$ \\ Daniele Chiappe, $\dagger^{a}$ Eugenio Cinquanta, (iD c Christian Martella, (D) a \\ Emiliano Bonera, ${ }^{\mathrm{b}}$ Emilio Scalise (iD ${ }^{\mathrm{b}}$ and Carlo Grazianetti (D) ${ }^{\mathrm{a}}$
}

Received 14th November 2019, Accepted 23rd December 2019

DOI: $10.1039 / c 9 f d 00121 b$

In the realm of two-dimensional material frameworks, single-element graphene-like lattices, known as Xenes, pose several issues concerning their environmental stability, with implications for their use in technology transfer to a device layout. In this Discussion, we scrutinize the chemical reactivity of epitaxial silicene, taken as a case in point, in oxygen-rich environments. The oxidation of silicene is detailed by means of a photoemission spectroscopy study upon carefully dosing molecular oxygen under vacuum and subsequent exposure to ambient conditions, showing different chemical reactivity. We therefore propose a sequential $\mathrm{Al}_{2} \mathrm{O}_{3}$ encapsulation of silicene as a solution to face degradation, proving its effectiveness by virtue of the interaction between silicene and a silver substrate. Based on this method, we generalize our encapsulation scheme to a large number of metal-supported Xenes by taking into account the case of epitaxial phosphorene-on-gold.

\section{Introduction}

Epitaxial Xenes are a quickly emerging reality in the realm of two-dimensional (2D) crystals beyond graphene. They were recently sorted into two generations according to the position of the constitutive element (X) in the periodic table (the first generation includes elements in the silicon group, whereas the second includes elements in the boron, nitrogen, and oxygen groups). ${ }^{1}$ Compared to other 2D players, they constitute a broad portfolio of materials with variable and configurable properties depending on the growth environment (e.g. the substrate) or the atomistic details (e.g. buckled bonding). As epitaxial materials, they are scalable and thus look promising for industrial applications. The heavier among

${ }^{a}$ CNR-IMM, Unit of Agrate Brianza, Via C. Olivetti 2, 20864 Agrate Brianza, Italy. E-mail: alessandro.molle@ cnr.it

${ }^{b}$ Dipartimento di Scienza dei Materiali, Università degli Studi Milano-Bicocca, Via R. Cozzi 55, 20125, Milano, Italy

${ }^{c}$ CNR-IFN, Piazza Leonardo da Vinci 32, 20133 Milano, Italy

$\dagger$ Present address: ASM Microchemistry, Pietari Kalmin katu 3, 00560 Helsinki (FI). 
them are also prone to hosting a $2 \mathrm{D}$ topological insulating state of matter. ${ }^{2,3}$ While structure and electronic properties may vary throughout the Xene generations, ${ }^{\mathbf{4 , 5}}$ a common issue for all single-layer Xenes relates to stability against environmental reactivity. This is basically due to the fact that bonding in Xenes is less stable than in graphene or other layered compounds. A paradigmatic example in this respect is silicene (and silicene-like materials like germanene and stanene), where pseudo $\mathrm{sp}^{2}$ bonding is artificially induced by means of configurational constraints. $^{6}$ This synthetic set-up poses several questions concerning the stability of silicene and similar materials. For instance, is silicene prone to degradation under ambient conditions? How does silicene undergo oxidation? How to protect silicene from degradation? And how to extend such a protective process to the whole class of Xenes? In this Discussion, we elaborate on specific aspects of the chemical stability of the Xenes by reporting original data on the exposure of epitaxial silicene to a $\mathrm{O}_{2}$-rich environment in ultra-high vacuum (UHV) growth conditions. Silicene, as well as other Xenes, undergoes degradation in environmental conditions unless a protective layer is applied. Encapsulation, as opposed to passivation, aims to form a barrier to the external atmosphere without changing either the bonding or the functional properties of the Xenes. This can be a difficult task because we need the encapsulating layer to grow on top of the Xene, while having minimal interaction with it. In this respect, we state the conditions for a universal encapsulation scheme for metal-supported Xenes and support it with a preliminary ab initio model. To corroborate the universal character of the approach, we scrutinize the epitaxial phosphorene on $\mathrm{Au}(111)$ as a second case in point.

\section{Silicene reactivity}

The oxidation of silicon constituted a milestone in integrated microelectronic circuits, enabling the ubiquitous integration of silicon. Now that silicon-based devices are approaching ultimate scaling, the oxidation of silicene, namely silicon at the 2D level, may open new technology paths of silicon exploitation in nanoelectronics. One step in this direction is to investigate how the chemical bonding in epitaxial silicene is affected by an oxygen-rich environment. We elucidate this scenario in a variable dosage experiment starting from an UHV condition and monitoring the synchrotron radiation Si 2p core-level photoemission line of epitaxial silicene-on-silver(111) with increasing $\mathrm{O}_{2}$ partial dose. Two silicene configurations were taken into account, the low-temperature mixed $4 \times$ $4 / \sqrt{ } 13 \times \sqrt{ } 13$ phase (Fig. 1a) and the high-temperature $2 \sqrt{ } 3 \times 2 \sqrt{ } 3$ phase (Fig. 1b), as they were proven to be the precursors for a room temperature operational field effect transistor. ${ }^{7}$ Basically, the two configurations differ in terms of their buckled bond pattern, ${ }^{8}$ as evidenced by the markedly different positioning of the $\mathrm{E}_{2 \mathrm{~g}}$ Raman mode as well as lower frequency features. ${ }^{9}$ For each configuration, Si $2 \mathrm{p}$ spectra are reported for the freshly grown silicene, after $75 \mathrm{~L}$ and $750 \mathrm{~L} \mathrm{O}_{2}$ exposure. The Si 2 p lines of the freshly grown silicene phases display a multipeak shape profile that was previously rationalized as coming from the interplay of three different elemental ( $\mathrm{Si}-\mathrm{Si}$ ) bonding environments termed $\mathrm{Si}^{\alpha}, \mathrm{Si}^{\beta}$, and $\mathrm{Si}^{\gamma} .{ }^{\mathbf{1 0}}$ Similar to Fleurence et al.,$^{11}$ these components reflect different positioning of the $\mathrm{Si}$ atoms with respect to the $\mathrm{Ag}$ surface, namely hollow sites, intermediate sites, and coincidence sites. This substrate sensitivity was also observed in a position- 

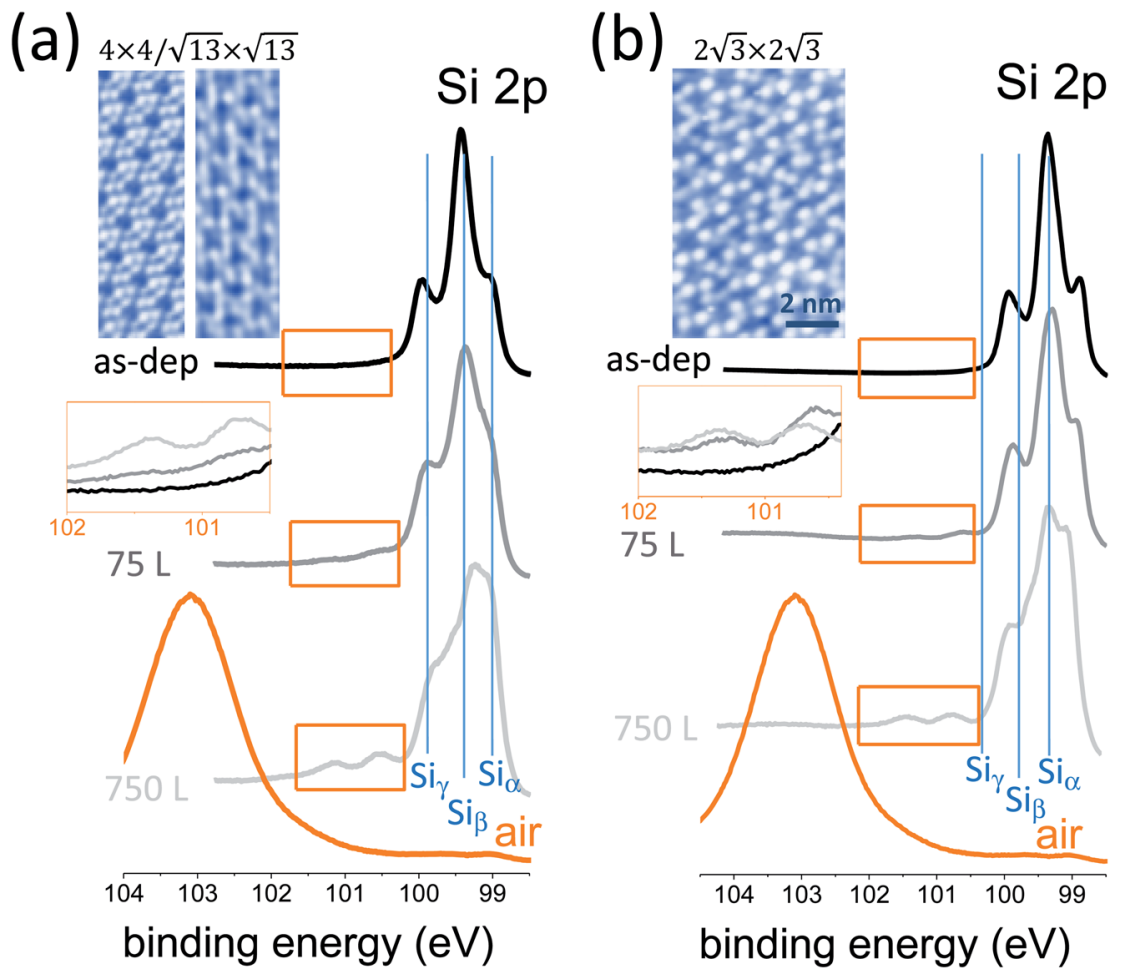

Fig. 1 Si $2 p$ core level photoemission lines (from top to bottom) for freshly grown siliceneon-silver, after $75 \mathrm{~L} \mathrm{O}_{2}$ exposure, after $750 \mathrm{~L} \mathrm{O}_{2}$ exposure, and after exposure to air. Core level lines are reported for two different silicene configurations, the $4 \times 4 / \sqrt{ } 13 \times \sqrt{ } 13$ mixed phase (a) and the $2 \sqrt{3} \times 2 \sqrt{3}$ phase (b). Images at the top of each panel are scanning tunneling topographies of the two configurations. Insets in both panels (intermediate position on the left side of the panels) are magnifications of a limited range of the Si $2 p$ lines in order to elucidate the $\mathrm{O}_{2}$-induced oxidation of silicene.

dependent local density of states. ${ }^{12}$ Limited oxidation takes place when the $\mathrm{O}_{2}$ dosage is varied from 75 to $750 \mathrm{~L}$, as may be deduced from the emergence of a minor feature that is characteristic of $\mathrm{Si}^{4+}$ bonding in $\mathrm{SiO}_{2}$ (see also the magnification of the spectral region in the insets). Conversely, the Si 2 p profile dramatically changes in the proportion of its elemental components. This fact is apparent from the evolution of the Si 2 p line as a function of the $\mathrm{O}_{2}$ dosage in both silicene configurations. This aspect relies on substantial variation of the bonding population inside each individual silicene phase as a consequence of oxygen exposure. In other words, silicene reacts to $\mathrm{O}_{2}$ exposure by rebalancing its elemental bonding components with respect to its pristine form, while $\mathrm{SiO}_{2}$ bonding formation is quite limited. In detail, the $\mathrm{Si}^{\alpha}$ component corresponding to hollow sites of silicon atoms undergoes a relative increase with increased $\mathrm{O}_{2}$ exposure from 75 to $750 \mathrm{~L}$. Qualitatively, this behaviour looks like a silicene lattice rearrangement, with silicon atoms preferentially positioned far from the underlying $\mathrm{Ag}$ atoms as if this recombination could diminish the interaction of silicene with the substrate. This picture can be related to previously reported $\mathrm{O}_{2}$ intercalation in the silicon bilayer. ${ }^{13}$ If this is the case, an open question is how post- 
growth $\mathrm{O}_{2}$ exposure can be exploited to disentangle silicene from its substrate without corrupting its elemental lattice, with a substantial benefit in the transfer of silicene into a device substrate. ${ }^{7}$

Ultimately, if silicene is exposed to air, complete oxidation to a $\mathrm{SiO}_{2}$ compound takes place, as follows from the emergence of a broad peak at $103.50 \mathrm{eV}$ corresponding to the $\mathrm{Si}^{4+}$ valence state in Si bonding. In this latter stage, it is not clear at the moment whether this silicene-derived $\mathrm{SiO}_{2}$ is recast as a $2 \mathrm{D}$ oxide layer (conformal to the original silicene) or as a clustered adsorbate. Open questions remain as to what kind of oxidation may take place when considering multilayer silicene as an alternative configuration to conventional cubic-structured silicon, which may unravel new mechanisms for silicon oxidation. More specifically, we wonder whether silicene can be uniformly oxidized so as to form a $2 \mathrm{D}$ silicon oxide either in a single layer or in a multilayer state. If so, we also wonder to what extent this silicene oxide would differ from the $2 \mathrm{D}$ silicon oxide frameworks grown on metal surfaces as reported by Büchner et al. ${ }^{14}$ This is an interesting set of questions aimed at the development of a new type of $2 \mathrm{D}$ insulator.

\section{Encapsulation of silicene}

\section{The experimental framework}

The environmental oxidation of silicene is a hurdle for its usage in applications. Methods to save silicene from degradation have been in demand since the early stages of silicene's debut. One possible effective option turned out to be $\mathrm{Al}_{2} \mathrm{O}_{3}$ encapsulation sequentially carried out in UHV conditions after silicene growth by means of reactive co-deposition of an atomic $\mathrm{Al}$ beam with $\mathrm{O}_{2}$ molecules. ${ }^{15}$ This methodology results in the preservation of the chemical environment of silicene by means of a protective $\mathrm{Al}_{2} \mathrm{O}_{3}$ layer on silicene by sequentially growing elemental $\mathrm{Al}$ in the first stage, and $\mathrm{Al}$ in an $\mathrm{O}_{2}$-rich environment in the second stage. The initial elemental Al prevents silicon from oxidation when inletting $\mathrm{O}_{2}$ gas in the second stage, so as to have an overall $\mathrm{Al}_{2} \mathrm{O}_{3}$ layer at the end. No chemical interaction occurs between silicene and $\mathrm{Al}_{2} \mathrm{O}_{3}$, as demonstrated by the unaltered shape
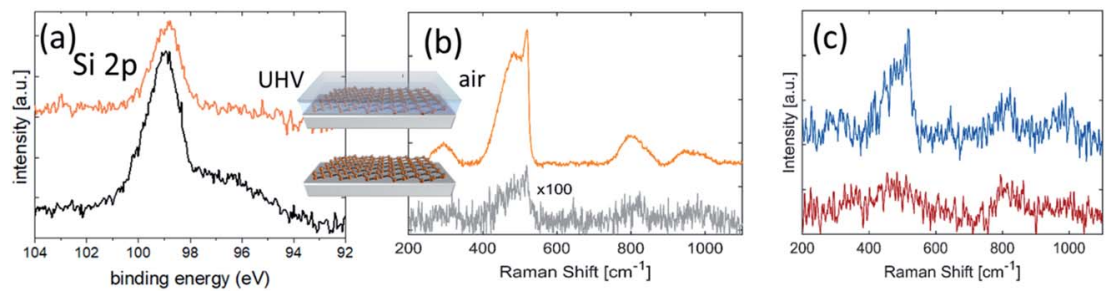

Fig. 2 (a) Si $2 p$ core-level photoemission lines taken on freshly grown silicene-on-silver (bottom) and after $\mathrm{Al}_{2} \mathrm{O}_{3}$ encapsulation (top). The right-side feature in the former spectrum is related to $\mathrm{Ag} 4 \mathrm{~s}$ photoemission from the substrate, and is no longer detected in the encapsulated silicene owing to the $\mathrm{Al}_{2} \mathrm{O}_{3}$ layer thickness ( $\sim 5 \mathrm{~nm}$ ); (b) Raman spectra of (top) capped and (bottom) uncapped silicene/Ag(111). In both cases the samples are exposed to air when recording the Raman spectrum and the silicene lattice is preserved only in the case of the encapsulated sample; (c) Raman spectra of unprotected silicene/ $\mathrm{Ag}(111)$ obtained after 1 minute (top: same as the bottom spectrum in panel (b)) and $\sim 5$ minutes after air exposure (bottom). The spectra are vertically stacked for clarity. 
profile of the Si 2p core level photoemission line in Fig. 2a prior to and after $\mathrm{Al}_{2} \mathrm{O}_{3}$ deposition. An extra Ag $4 \mathrm{~s}$ feature from the substrate does not appear in the $\mathrm{Si} 2 \mathrm{p}$ line of the pristine silicene after encapsulation, owing to the added $\mathrm{Al}_{2} \mathrm{O}_{3}$ thickness and consequent reduced substrate sensitivity in the photoemission. The $\mathrm{Al}_{2} \mathrm{O}_{3}$ encapsulation presents several benefits. It is a sequential process with silicene epitaxy within the same UHV environment. As such, it is not affected by collateral contamination. It works as an insulating layer for applications where a gate is needed. The effectiveness of the $\mathrm{Al}_{2} \mathrm{O}_{3}$ encapsulation is phenomenological evidence substantiated by in situ X-ray photoelectron spectroscopy (XPS) and ex situ Raman spectroscopy, as reported in Fig. 2.

As an identification probe, Raman spectroscopy has generally proved to be a superior tool to identify graphene and graphene-related materials. ${ }^{16}$ It was used for silicene too in order to check the silicene integrity after encapsulation, as reported in Fig. 2a. This is demonstrated in the comparative Raman spectroscopy study in Fig. 2b. In detail, Fig. 2b compares the encapsulated silicene (in air) with uncapped silicene in the early stage of degradation upon exposure to ambient air. The spectrum of silicene-on-silver(111) acquired just after its exposure to ambient conditions shows a feature around $500 \mathrm{~cm}^{-1}$, composed of a prominent narrow peak at $516 \mathrm{~cm}^{-1}$, plus a broad shoulder on the lower energy side $\left(\sim 460 \mathrm{~cm}^{-1}\right)$. These features are comparable with the Raman active modes of silicene-onsilver(111) substrates maintained in UHV conditions ${ }^{17}$ and, together with the band at $800 \mathrm{~cm}^{-1}$, are indicative of the presence of a partially oxidized ordered $\mathrm{SiO}_{2}$ phase. ${ }^{18}$ More detailed characterization of the Raman spectrum of silicene is extensively reported elsewhere. ${ }^{9}$ Here we emphasize the effectiveness of encapsulation in preserving silicene from degradation upon direct exposure. Nonetheless, fast degradation occurs for both silicene configurations after exposure to air if uncapped, as seen from the XPS spectra reported in Fig. 1. The dynamics of silicene oxidation are so fast that close real-time monitoring of silicene evolution is necessary to unveil the mechanism of $\mathrm{Si}$ reactivity under environmental conditions. As uncapped silicene is extracted from the growth chamber and brought into ambient lab conditions, the reaction with $\mathrm{O}_{2}$ occurs immediately, as evidenced by the Raman spectrum reported in Fig. 2c. Sizable modifications affect the Raman spectrum as a function of the exposure time. The disappearance of the sharp peak is accompanied by the broadening of the feature at $500 \mathrm{~cm}^{-1}$, whose intensity becomes comparable with the feature at $800 \mathrm{~cm}^{-1}$. These two observations may reflect the complete amorphization of the $\mathrm{SiO}_{2}$ phase resulting from early exposure, thus confirming the recent results obtained by means of in situ Raman spectroscopy at a low $\mathrm{O}_{2}$ dose. ${ }^{19}$ The dynamics of the bare silicene/Ag(111) exposed to ambient conditions are clearly dominated by the reaction with $\mathrm{O}_{2}$. The overall process could be depicted as a two-step reaction. The early interaction of the silicene layer with $\mathrm{O}_{2}$ leads to the formation of partially ordered $\mathrm{SiO}_{2}$ phase clusters with a small portion of silicene in its original structure. As the process progresses, the system evolves towards the amorphous phase of thin clusterassembled $\mathrm{SiO}_{x}$. To confirm this two-step picture and describe the silicene degradation process, in situ monitoring of the structural properties of the system as a function of the $\mathrm{O}_{2}$ dose would help to disentangle the two proposed kinetics. The initial tendency of the silicene layer to preserve its order may be related to the interaction with the metallic substrate that makes the $\mathrm{Si}$ atoms less prone to oxidation. 
Overall, a question arises regarding which mechanism favours $\mathrm{Al}_{2} \mathrm{O}_{3}$ accommodation on silicene-on-Ag without apparently affecting its structure.

\section{A theoretical framework}

An argument to support the picture as outlined above comes from preliminary density functional theory (DFT) simulations of silicene reactivity towards $\mathrm{Al}$ atom adsorption first, and $\mathrm{Al}_{2} \mathrm{O}_{3}$ accommodation second. We propose simulations for two different configurations, free-standing silicene and silicene-on-Ag. In order to further investigate this mechanism, a preliminary ab initio investigation of the interaction between $\mathrm{Al}$ or $\mathrm{O}_{2}$ and a $4 \times 4$ reconstruction of silicene on $\mathrm{Ag}(111)$ has been performed by means of DFT calculations detailed in the methods section. The results of the structural relaxations are summarized in Fig. 3, where two relevant aspects can be highlighted. First, an $\mathrm{Al}$ atom (light blue sphere) turns out to be favorably incorporated into the crystal lattice of free-standing silicene and it strongly affects the silicene structure (blue sphere lattice) after relaxation (Fig. 3a), whereas the same atom on Ag-supported silicene (Fig. 3b) tends to move apart from the Si surface upon relaxation. Hence, one can infer that the silicene layer is
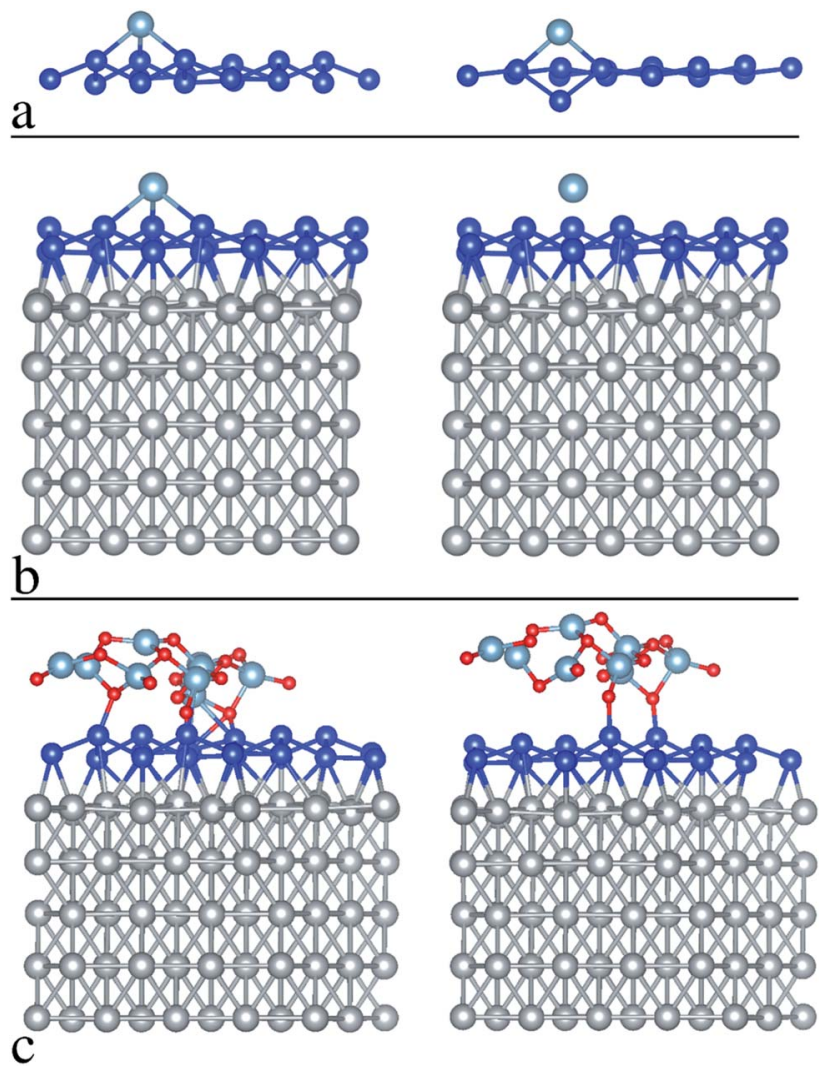

Fig. 3 DFT simulation before relaxation (left) and after relaxation (right) in the case of: (a) Al atom absorption to free-standing silicene, (b) Al atom absorption to silicene-on-silver, (c) $\mathrm{Al}_{2} \mathrm{O}_{3}$ accommodation on silicene-on-silver. 
chemically "protected" by the hybridization of the Si atoms with the Ag atoms of the substrate, and in fact the calculated binding energy of the $\mathrm{Al}$ atom on the silicene layer is reduced by about $0.25 \mathrm{eV}$ in the presence of the $\mathrm{Ag}$ substrate, as compared to the free-standing case. It should be noted that this is tendential behavior for an individual $\mathrm{Al}$ adatom accommodated on the silicene face that can hardly be matched with the collective behavior of an Al layer grown on silicene. Nonetheless, this individual picture gives us an idea of the tendential influence of the $\mathrm{Ag}$ substrate on silicene reactivity during $\mathrm{Al}_{2} \mathrm{O}_{3}$ deposition. In the latter case, the "lower reactivity" of the Ag-supported silicene (compared to the free-standing case) leads the $\mathrm{O}_{2}$ molecules ( $\mathrm{O}$ atoms are depicted as red spheres in Fig. 3) to preferably bind with the $\mathrm{Al}$ atoms, resulting in a relaxed structure that only marginally influences the silicene bonds (see Fig. 3c, which illustrates the asgrown and relaxed $\mathrm{Al}_{2} \mathrm{O}_{3}$ on $\mathrm{Ag}$-supported silicene). It should be noted that this picture is only specific to the Ag-supported silicene and may fail in other cases, e.g. silicene on $\mathrm{ZrB}_{2}$, where the extent of hybridization between the silicene layer and the substrate can change. Although it was noted that effective encapsulation can be substrate-dependent, ${ }^{20}$ this preliminary outcome suggests a method to apply $\mathrm{Al}_{2} \mathrm{O}_{3}$ encapsulation to Xenes supported by noble metal surfaces.

Though preliminary, these data show that the $\mathrm{Al}_{2} \mathrm{O}_{3}$ capping protocol can be applied to epitaxial Xenes with an interaction with the substrate strong enough to inhibit or minimize chemisorption to $\mathrm{Al}$ adatoms. This usually happens on noble metal surfaces hosting an Xene layer, as in the case of silicene-on-silver, and should be further investigated for silicene deposition on sapphire. ${ }^{21}$ A question arises as to whether other Xenes of the same kind may benefit from such an encapsulation scheme. More generally, we wonder whether the encapsulation reported here can be taken as a universal methodology for Xenes on metal surfaces. In the following section we partially address this by taking into account the case of epitaxial phosphorene.

\section{Encapsulation of epitaxial phosphorene}

The strategy developed for the encapsulation of silicene on a metallic substrate has been fruitfully exploited to prevent the oxidation of exfoliated black phosphorous flakes on $\mathrm{SiO}_{2} / \mathrm{Si}$ substrate. ${ }^{22}$ The encapsulation thus also works for highly reactive van der Waals materials on inert substrates. Therefore, it is interesting to further explore its applicability to the vast family of $2 \mathrm{D}$ materials with a focus on epitaxial Xenes.

Epitaxial phosphorene is a good case for which to validate $\mathrm{Al}_{2} \mathrm{O}_{3}$ encapsulation for metal-supported Xenes beyond silicene-on-silver. Epitaxial phosphorene results from the vacuum evaporation of a $\mathrm{P}_{4}$ molecular flux that undergoes dissociative chemisorption when ground on an $\mathrm{Au}(111)$ surface. In the substrate temperature range $240-270{ }^{\circ} \mathrm{C}$, chemisorbed phosphorus molecular species selfarrange in a flower-like honeycomb pattern with hexagonal symmetry, as shown in the atomically resolved scanning tunneling microscopy (STM) topography in Fig. 4a. We name this structure epitaxial phosphorene to discriminate it from a single layer of black phosphorus, conventionally termed phosphorene. In contrast to silicene, epitaxial phosphorene possesses a local semiconducting character $^{23,24}$ and completely covers the Au terraces with a single superstructure (Fig. 4b). The observed morphology was originally attributed to an epilayer with 
a)

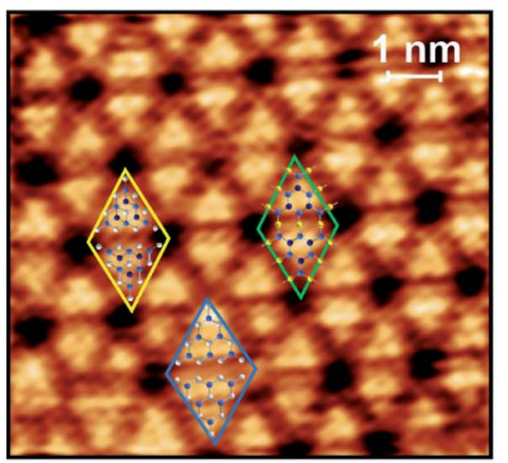

C)

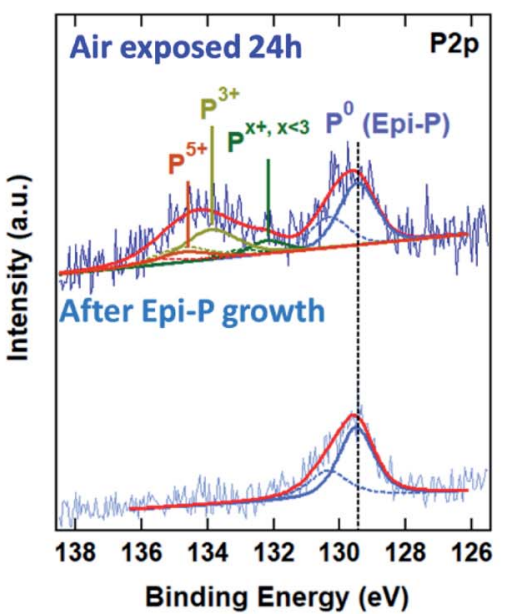

b)

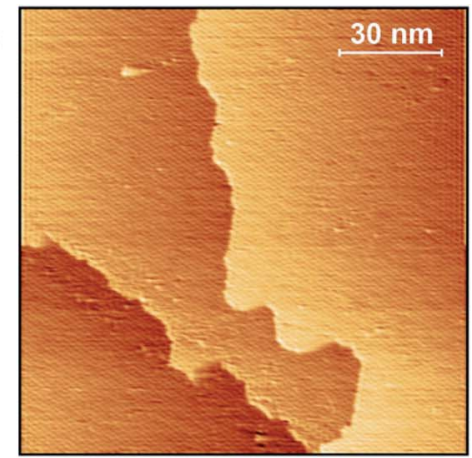

d)

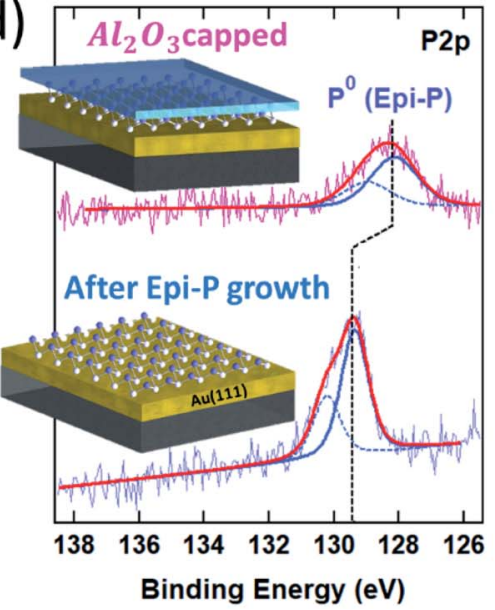

Fig. 4 (a) High-resolution $8 \times 8 \mathrm{~nm}^{2}$ STM image of epitaxial phosphorene. The rhombi are $(5 \times 5)$ unit cells enclosing local atomic models proposed for epitaxial phosphorene: the $\mathrm{P}_{16}$ blue-phosphorene structure (blue), the "adatom-rich" structure (yellow) and the recently proposed Au-decorated phosphorene network (green). In the overlapping models $\mathrm{P}$ atoms are represented by white, light blue and dark blue spheres according to the distance from the substrate, and Au atoms by yellow spheres. (b) Large-scale $130 \mathrm{~nm}$ $\times 130 \mathrm{~nm}^{2}$ STM image of epitaxial phosphorene; (c) comparative XPS study of the P $2 p$ line of freshly grown epitaxial phosphorene (bottom) and after exposure to air (top); (d) comparative XPS study of the P $2 p$ line of freshly grown epitaxial phosphorene (bottom) and after $\mathrm{Al}_{2} \mathrm{O}_{3}$ encapsulation (top).

the same buckled structure of the theoretically predicted free-standing bluephosphorene lattice. In this model all the petals of the flower-like pattern correspond to $\mathrm{P}_{16}$ blue-phosphorene islands matching half of the $5 \times 5 \mathrm{Au}(111)$ unit cell. ${ }^{23-25}$ However, close inspection of the high-resolution STM image in Fig. 4a reveals that only a few petals show six bright protrusions typical of a $\mathrm{P}_{16}$ bluephosphorene island, while most of them consist of three bright protrusions. This has been explained by introducing a model where two local atomic configurations may coexist together. ${ }^{26,27}$ Beside sparse $\mathrm{P}_{16}$ blue-phosphorene islands (see the blue rhombus in Fig. 4a), "adatom-rich" structures are also possible. In the "adatom-rich" structure three additional phosphorus atoms are accommodated on the top layer (see the yellow rhombus in Fig. 4a). More recently, a new 
interpretation of the structure of epitaxial phosphorene questioned all the previously discussed atomic models. As reported in ref. 28, the structure of epitaxial phosphorene may correspond to an Au-decorated phosphorene network rather than an epilayer. According to this model, the triangular petals coincide with buckled blue-phosphorene $\mathrm{P}_{9}$ monomers (with the top three $\mathrm{P}$ atoms protruding at a larger distance from the Au substrate) linked to three other $\mathrm{P}_{9}$ monomers on their sides by gold adatoms coming from the substrate (see the green rhombus in Fig. 4a).

Like silicene (but to a different extent), epitaxial phoshorene is subject to oxidation when exposed to air. Its stability is elucidated in the comparative XPS study in Fig. 4c, where the P 2p core level photoemission line is reported for freshly grown epitaxial phosphorene (bottom) and after exposure to air (top). As can be noted from the permanence of the elementary $\mathrm{P}-\mathrm{P}$ bonding component (denoted $\mathrm{P}^{0}$ in Fig. 4c), at $129.4 \mathrm{eV}$ epitaxial phosphorene still survives after air exposure, in marked contrast to silicene. The photoemitted P 2p core electrons of air-exposed epitaxial phosphorene show a broad oxide peak from 132 to $136 \mathrm{eV}$. By fitting this oxide peak through spin-orbit doublets with a separation of $0.86 \mathrm{eV}$ and a fixed $2: 1$ intensity ratio, the resulting components at $134.7 \mathrm{eV}$ and $133.8 \mathrm{eV}$ can be attributed to phosphorus oxidation states of +5 and +3 , respectively, whereas the component at $132.1 \mathrm{eV}$ can be assigned to lower oxidation states. Thus, the oxidation of epitaxial phosphorene in air may involve the formation of $\mathrm{P}_{2} \mathrm{O}_{5}$ and $\mathrm{P}_{2} \mathrm{O}_{3}$ oxides and $\mathrm{P}_{2} \mathrm{O}_{x, x<3}$ suboxides, similar to what has been reported for air-exposed black phosphorus films. ${ }^{29,30}$ Oxygen is known to play an important role in the preliminary stages of black phosphorus oxidation, ${ }^{31}$ but alone does not lead to a complete physical degradation of the material. Short-term exposure to high-purity $\mathrm{O}_{2}$ has been shown to oxidize few-layer black phosphorus at a much slower rate than the combined action of oxygen and water producing low oxidation states. ${ }^{31}$ Similarly, epitaxial phosphorene shows a remarkably high oxidation endurance to limited molecular oxygen dosing in UHV. ${ }^{26}$ At higher doses, oxygen atoms preferentially absorb on top of one of the protruding phosphorus trimers without degrading the epitaxial phosphorene superstructure. ${ }^{32}$ These facts reveal a peculiar oxidation chemistry of epitaxial phosphorene that differs from that of silicene and shares some similarities with the oxidation mechanism of black phosphorus. Accurate investigations are needed to disentangle the oxidation scheme of epitaxial phosphorene and resolve open questions such as the chemistry of epitaxial phosphorene with water, alone or in combination with another oxidizing agent like oxygen, as well as the influence of exposure to light.

The effect of the $\mathrm{Al}_{2} \mathrm{O}_{3}$ encapsulation of epitaxial phosphorene is elucidated in Fig. $4 \mathrm{~d}$ by comparing the $\mathrm{P} 2 \mathrm{p}$ line prior to and after $\mathrm{Al}_{2} \mathrm{O}_{3}$ capping. Despite the reduced photoemission intensity (due to the extra thickness of the $\mathrm{Al}_{2} \mathrm{O}_{3}$ top layer), the shape profile of the $\mathrm{P} 2 \mathrm{p}$ line shows no substantial change except for a $-1.2 \mathrm{eV}$ shift of the binding energy between the two lines (to a lesser extent a similar shift is also observed for silicene on $\mathrm{Ag}$ ). The former fact validates the encapsulating action of the $\mathrm{Al}_{2} \mathrm{O}_{3}$ layer and positions $\mathrm{Al}_{2} \mathrm{O}_{3}$ encapsulation as a candidate for a general approach to the stabilization of Xenes supported on metal substrates. The latter fact stems from the peak positioning with reference to the $\mathrm{Au}$ 4f peaks (binding energy positioning of the core level peaks is calibrated using the $\mathrm{Au} 4 \mathrm{f}$ position as usual, for each process stage). Shifts in the binding energies can be due to charge incorporation causing the Fermi level to move away 
from its pristine position in the uncapped layer. We interpret this behaviour as being due to an interface dipole formation around the phosphorene environment, according to which fixed charges intrinsic to the $\mathrm{Al}_{2} \mathrm{O}_{3}$ film are compensated by an image charge in the Au/phosphorene superstructure. ${ }^{33,34}$ This picture is corroborated by the observation that the original binding energy is restored whenever the $\mathrm{Al}_{2} \mathrm{O}_{3}$ capped phosphorene is exposed to air (data not shown), as far as the environmental reactivity may induce the saturation of charged defects in $\mathrm{Al}_{2} \mathrm{O}_{3}$ and a reset of the electrical balance between the stacks.

Oxidation of epitaxial phosphorene is a key aspect to understand in order to develop processing protocols such as layer transfer aiming at device fabrication. Recently, it was shown that $\mathrm{Al}_{2} \mathrm{O}_{3}$ encapsulation can save epitaxial phosphorene from complete degradation in a wet environment (used for substrate etching). ${ }^{26}$ Nonetheless, partial oxidation takes place and it is not clear at the moment what the relevant reactivity path is. This will open a framework to engineer processes enabling the full exploitation of large-scale phosphorus at the $2 \mathrm{D}$ level, thus bypassing the current size limitation of black phosphorus flakes.

\section{Summary}

Xenes are a new frontier in the physics and chemistry of 2D materials. However, their stability in environmental conditions is a hurdle for applications. This Discussion provides a playground to explore several aspects of the chemical reactivity of Xenes and to develop a general methodology to prevent the environmental degradation of Xenes out of vacuum. The general character of the Discussion is to propose data inspiring open questions or issues yet to be solved. In detail, the first aspect considered is the chemical reactivity upon $\mathrm{O}_{2}$ exposure and in air. This is showcased in the paradigmatic epitaxial silicene-on-silver by means of photoemission and Raman spectroscopy investigations. Second, a solution to the fast degradation of silicene is proposed, which consists of the sequential encapsulation of silicene with an $\mathrm{Al}_{2} \mathrm{O}_{3}$ capping layer. The effectiveness of the approach is substantiated and supported by a preliminary DFT model suggesting that the silicene-silver interaction is a key mechanism to save silicene integrity after $\mathrm{Al}_{2} \mathrm{O}_{3}$ deposition. This idea is further developed for epitaxial phosphorene-on-gold in order to validate the universality of the approach for the Xene-on-metal framework. In the latter case, the encapsulation works as effective protection and, due to the peculiar charge exchange at the $\mathrm{Al}_{2} \mathrm{O}_{3}$-phosphorene interface, dipole-induced electronic band bending is reported as an effect of the heterostructure. Additional Xenes on metal substrates will represent promising tests to unravel possible universal oxidation mechanisms as well as to develop encapsulation processes.

\section{Methods}

\section{Sample growth}

Epitaxial growth of Xenes was performed in UHV conditions (base pressure $10^{-10}$ mbar) in a temperature range of $210-290{ }^{\circ} \mathrm{C}$ by means of molecular flux released from e-beam evaporators or effusion cells on metal (Ag, $\mathrm{Au}$ ) film surfaces supported by mica substrates and prepared by several cycles of $\mathrm{Ar}^{+}(1 \mathrm{keV})$ sputtering and annealing at $500{ }^{\circ} \mathrm{C}$. Oxygen dosage experiments were performed in the UHV 
environment by exposing the silicene surface to an $\mathrm{O}_{2}$-rich atmosphere $(100 \mathrm{~s}$ and $1000 \mathrm{~s}$ at $1 \times 10^{-6}$ mbar to get 75 and $750 \mathrm{~L}$, respectively).

\section{Experimental characterization}

Synchrotron radiation core-level photoemission spectroscopy in Fig. 1 was performed at the VUV-beamline at the Elettra Synchrotron Radiation facility in Italy, with the electron spectrometers placed at $45^{\circ}$ with respect to the direction of the horizontally polarized photon beam and with a photon energy $h \nu=130 \mathrm{eV}$; XPS data in Fig. 2 and 4 were acquired by means of a non-monochromatic $\mathrm{Mg} \mathrm{K} \alpha$ source $(1253.6 \mathrm{eV})$ and their energy positioning was calibrated by taking the tabulated $\mathrm{Ag}$ and Au core-level positions as references for the case of silicene and phosphorene, respectively; Raman spectroscopy in Fig. 2 was performed with a single $60 \mathrm{~s}$ long acquisition by exploiting an Invia Renishaw spectrometer equipped with the $514.5 \mathrm{~nm}$ wavelength of an $\mathrm{Ar}^{+}$laser Raman spectrum; STM topographies in Fig. 1 and 4 were acquired at room temperature with an Omicron scanning tunneling microscope (STM) equipped with a homemade chemically etched tungsten tip.

\section{DFT modelling details}

$A b$ initio simulations are performed by exploiting DFT with PBE exchangecorrelation potential in a generalized gradient approximation (GGA) ${ }^{35}$ and using a non-local functional for the van der Waals forces, ${ }^{36}$ as implemented in Quantum Espresso. ${ }^{37}$ We use a plane-wave basis set with a kinetic energy cutoff of 40 Ry and the $\Gamma$ point for sampling the Brillouin zone of the simulated supercell. The silicene/Ag system has been modeled by a supercell containing $5 \mathrm{Ag}$ (111) atomic layer with the silicene layer on top. The supercell is periodic in the $x-y$ directions, while $15 \AA$ of vacuum has been exploited to avoid interactions between periodic replica in the $z$ direction and a dipole correction has been also used. A single $\mathrm{Al}$ atom, an $\mathrm{O}_{2}$ molecule, or an $\mathrm{Al}_{2} \mathrm{O}_{12}$ cluster has been inserted on the silicene surface at an initial distance that ensures the formation of bonds between the $\mathrm{Si}$ atoms of the silicene layer and the $\mathrm{Al}$ or $\mathrm{O}$ atoms on top. Then structural relaxations have been performed until the average atomic force was lower than $10^{-4} \mathrm{Ry}$ per Bohr, by using a quasi-Newton algorithm, both including the Ag substrate and considering a bare free-standing silicene layer.

\section{Conflicts of interest}

There are no conflicts to declare.

\section{Acknowledgements}

The authors acknowledge P. Moras, S. Sanjoy, and C. Carbone (CNR-ISM, Trieste, Italy) for support with the synchrotron measurements. A. M., C. M. and C. G. acknowledge financial support from H2020 Framework Programme funding, call ERC CoG 2017 Grant N. 772261 "XFab”.

\section{Notes and references}

1 C. Grazianetti, C. Martella and A. Molle, Phys. Status Solidi RRL, 2019, 1900439. 
2 A. Molle, J. Goldberger, M. Houssa, Y. Xu, S. C. Zhang and D. Akinwande, Nat. Mater., 2017, 16, 163-169.

3 Y. Xu, B. Yan, H.-J. Zhang, J. Wang, G. Xu, P. Tang, W. Duan and S.-C. Zhang, Phys. Rev. Lett., 2013, 111, 136804.

4 A. J. Mannix, B. Kiraly, M. C. Hersam and N. P. Guisinger, Nat. Rev. Chem., 2017, 1, 0014.

5 Y. Yamada-Takamura and R. Friedlein, Sci. Technol. Adv. Mater., 2014, 15, 064404.

6 A. Molle, C. Grazianetti, L. Tao, D. Taneja, M. H. Alam and D. Akinwande, Chem. Soc. Rev., 2018, 47, 6370-6387.

7 L. Tao, E. Cinquanta, D. Chiappe, C. Grazianetti, M. Fanciulli, M. Dubey, A. Molle and D. Akinwande, Nat. Nanotechnol., 2015, 10, 227-231.

8 C. Grazianetti, D. Chiappe, E. Cinquanta, G. Tallarida and M. Fanciulli, Appl. Surf. Sci., 2014, 291, 109-112.

9 E. Cinquanta, E. Scalise, D. Chiappe, C. Grazianetti, B. Van Den Broek, M. Houssa, M. Fanciulli and A. Molle, J. Phys. Chem. C, 2013, 117, 16719.

10 J. Avila, P. De Padova, S. Cho, I. Colambo, S. Lorcy, C. Quaresima, P. Vogt, a. Resta, G. Le Lay and M. C. Asensio, J. Phys.: Condens. Matter, 2013, 25, 262001.

11 A. Fleurence, R. Friedlein, T. Ozaki, H. Kawai, Y. Wang and Y. YamadaTakamura, Phys. Rev. Lett., 2012, 108, 245501.

12 D. Chiappe, C. Grazianetti, G. Tallarida, M. Fanciulli and A. Molle, Adv. Mater., 2012, 24, 5088-5093.

13 Y. Du, J. Zhuang, J. Wang, Z. Li, H. Liu, J. Zhao, X. Xu, H. Feng, L. Chen, K. Wu, X. Wang and S. X. Dou, Sci. Adv., 2016, 2, e1600067.

14 C. Büchner, Z.-J. Wang, K. M. Burson, M.-G. Willinger, M. Heyde, R. Schlögl and H.-J. Freund, ACS Nano, 2016, 10, 7982-7989.

15 A. Molle, C. Grazianetti, D. Chiappe, E. Cinquanta, E. Cianci, G. Tallarida and M. Fanciulli, Adv. Funct. Mater., 2013, 23, 4340-4344.

16 A. C. Ferrari and D. M. Basko, Nat. Nanotechnol., 2013, 8, 235-246.

17 D. Solonenko, O. D. Gordan, G. Le Lay, H. Şahin, S. Cahangirov, D. R. T. Zahn and P. Vogt, 2D Mater., 2016, 4, 015008.

18 F. L. Galeener, A. J. Leadbetter and M. W. Stringfellow, Phys. Rev. B, 1983, 27, 1052-1078.

19 D. Solonenko, O. Selyshchev, D. R. T. Zahn and P. Vogt, Phys. Status Solidi, 2019, 256, 1800432.

20 R. Friedlein, H. Van Bui, F. B. Wiggers, Y. Yamada-Takamura, A. Y. Kovalgin and M. P. de Jong, J. Chem. Phys., 2014, 140, 204705.

21 C. Grazianetti, S. De Rosa, C. Martella, P. Targa, D. Codegoni, P. Gori, O. Pulci, A. Molle and S. Lupi, Nano Lett., 2018, 18, 7124-7132.

22 J. D. Wood, S. A. Wells, D. Jariwala, K.-S. Chen, E. Cho, V. K. Sangwan, X. Liu, L. J. Lauhon, T. J. Marks and M. C. Hersam, Nano Lett., 2014, 14, 6964-6970.

23 J. L. Zhang, S. Zhao, C. Han, Z. Wang, S. Zhong, S. Sun, R. Guo, X. Zhou, C. D. Gu, K. Di Yuan, Z. Li and W. Chen, Nano Lett., 2016, 16, 4903-4908.

24 J. Zhuang, C. Liu, Q. Gao, Y. Liu, H. Feng, X. Xu, J. Wang, J. Zhao, S. X. Dou, Z. Hu and Y. Du, ACS Nano, 2018, 12, 5059-5065.

25 E. Golias, M. Krivenkov, A. Varykhalov, J. Sánchez-Barriga and O. Rader, Nano Lett., 2018, 18, 6672-6678. 
26 C. Grazianetti, G. Faraone, C. Martella, E. Bonera and A. Molle, Nanoscale, 2019, 11, 18232-18237.

27 W. Zhang, H. Enriquez, Y. Tong, A. Bendounan, A. Kara, A. P. Seitsonen, A. J. Mayne, G. Dujardin and H. Oughaddou, Small, 2018, 14, 1804066.

28 H. Tian, J.-Q. Zhang, W. Ho, J.-P. Xu, B. Xia, Y. Xia, J. Fan, H. Xu, M. Xie and S. Y. Tong, Matter, 2020, 2, 111-118.

29 K. L. Kuntz, R. A. Wells, J. Hu, T. Yang, B. Dong, H. Guo, A. H. Woomer, D. L. Druffel, A. Alabanza, D. Tománek and S. C. Warren, ACS Appl. Mater. Interfaces, 2017, 9, 9126-9135.

30 W. Luo, D. Y. Zemlyanov, C. A. Milligan, Y. Du, L. Yang, Y. Wu and P. D. Ye, Nanotechnology, 2016, 27, 434002.

31 A. Ziletti, A. Carvalho, D. K. Campbell, D. F. Coker and A. H. Castro Neto, Phys. Rev. Lett., 2015, 114, 046801.

32 J. L. Zhang, S. Zhao, M. Telychko, S. Sun, X. Lian, J. Su, A. Tadich, D. Qi, J. Zhuang, Y. Zheng, Z. Ma, C. Gu, Z. Hu, Y. Du, J. Lu, Z. Li and W. Chen, Nano Lett., 2019, 19, 5340-5346.

33 L. Kornblum, J. A. Rothschild, Y. Kauffmann, R. Brener and M. Eizenberg, Phys. Rev. B: Condens. Matter Mater. Phys., 2011, 84, 155317.

34 Y. Wu, H.-S. Tao, E. Garfunkel, T. E. Madey and N. D. Shinn, Surf. Sci., 1995, 336, 123-139.

35 J. P. Perdew, K. Burke and M. Ernzerhof, Phys. Rev. Lett., 1996, 77, 3865-3868.

36 M. Dion, H. Rydberg, E. Schröder, D. C. Langreth and B. I. Lundqvist, Phys. Rev. Lett., 2004, 92, 246401.

37 P. Giannozzi, S. Baroni, N. Bonini, M. Calandra, R. Car, C. Cavazzoni, D. Ceresoli, G. L. Chiarotti, M. Cococcioni, I. Dabo, A. Dal Corso, S. de Gironcoli, S. Fabris, G. Fratesi, R. Gebauer, U. Gerstmann, C. Gougoussis, A. Kokalj, M. Lazzeri, L. Martin-Samos, N. Marzari, F. Mauri, R. Mazzarello, S. Paolini, A. Pasquarello, L. Paulatto, C. Sbraccia, S. Scandolo, G. Sclauzero, A. P. Seitsonen, A. Smogunov, P. Umari and R. M. Wentzcovitch, J. Phys.: Condens. Matter, 2009, 21, 395502. 